\title{
Humanlike, Task-Specific Reaching and Grasping with Redundant Arms and Low-Complexity Hands
}

\author{
Minas V. Liarokapis, Member, IEEE, Aaron M. Dollar, Senior Member, IEEE \\ and Kostas J. Kyriakopoulos Member, IEEE
}

\begin{abstract}
In this paper, we propose a methodology for closedloop, humanlike, task-specific reaching and grasping with redundant robot arms and low-complexity robot hands. Human demonstrations are utilized in a learn by demonstration fashion, in order to map human to humanlike robot motion. Principal Components Analysis (PCA) is used to transform the humanlike robot motion in a low-dimensional manifold, where appropriate Navigation Function (NF) models are trained. A series of grasp quality measures, as well as task compatibility indexes are employed to guarantee robustness of the computed grasps and task specificity of goal robot configurations. The final scheme provides anthropomorphic robot motion, task-specific robot arm configurations and hand grasping postures, optimized fingertips placement on the object surface (that results to robust grasps) and guaranteed convergence to the desired goals. The position and geometry of the objects are considered a-priori known. The efficiency of the proposed methods is assessed with simulations and experiments that involve different robot arm hand systems. The proposed scheme can be useful for various Human Robot Interaction (HRI) applications.
\end{abstract}

Index Terms: Robot Grasping, Anthropomorphism, Navigation Functions, Human Robot Interaction

\section{INTRODUCTION}

Over the last years a lot of effort has been put into building fully-actuated, highly dexterous, multifingered robot hands. But these hands require multiple motors for the multiple Degrees of Freedom (DoF), sophisticated sensing elements and electronics, as well as complicated software and control laws. Thus, they have increased complexity, weight and cost. According to Ma et al [1] increasing system dexterity can be accomplished either by adding hand complexity or arm kinematic redundancy. In this paper, instead of using expensive, complicated, multifingered robot hands, we utilize lowcomplexity, compliant, underactuated robot hands [2]-[4] as end-effectors of redundant robot arm hand systems. In this respect, we are able to employ robot arm's redundancy to execute more complex tasks or to optimize arm hand system's performance for specific tasks.

This work has been partially supported by the NSF grant IIS-1317976 and the European Commission with the Integrated Project no. 248587, THE Hand Embodied, within the FP7-ICT-2009-4-2-1 program Cognitive Systems and Robotics.

M. V. Liarokapis and A. M. Dollar are with the Department of Mechanical Engineering and Materials Science, School of Engineering and Applied Science, Yale University, 9 Hillhouse Avenue, New Haven, CT, 06520, USA. (email: \{minas.liarokapis,aaron.dollar\}@yale.edu).

K. J. Kyriakopoulos is with the School of Mechanical Engineering, National Technical University of Athens, 9 Heroon Polytechniou Str, Athens, 15780. (email: kkyria@mail.ntua.gr).

978-1-4673-7509-2/15/\$31.00 2015 IEEE
Nowadays it's quite typical also for robots to operate in human-centric, dynamic environments. Thus, anthropomorphism of robot motion becomes a desirable attribute for 1) aesthetical/psychological reasons (humans prefer robots moving in a humanlike manner as they feel more comfortably interacting with them) and 2) for safety reasons (humans can more easily predict humanlike motions and compensate their activities avoiding possible injuries [5], [6]). In order to derive anthropomorphic robot motion, human demonstrations have to be acquired and appropriate transformations have to be defined that will facilitate mapping of human to humanlike robot motion.

Mapping of human to robot motion has been a challenging problem in the field of robotics. Over the past years, four different human to robot hand motion mapping methodologies have been proposed: fingertips mapping [7], [8], joint-to-joint mapping [9], functional pose mapping [10] and object specific mapping [11]. Regarding human to robot arm motion mapping, most of the previous studies focused on a forward-inverse kinematics approach [12]. Regarding anthropomorphism of robot motion, authors in [13] used a criterion from ergonomics to extract human-like goal configurations for robotic arm hand systems, while in [14] they employed a series of bio-inspired optimization principles (e.g., minimization of hand jerk) to compute humanlike robot trajectories. Recently, we proposed a mapping scheme based on the analytic computation of inverse kinematics of a redundant robot arm, introducing the notion of functional anthropomorphism. Functional anthropomorphism has as first priority to guarantee the execution of specific human-imposed task goals (e.g., same position and orientation for human and robot wrists) and then to optimize anthropomorphism of robot configurations [15].

In everyday life grasping and manipulation tasks humans adopt postures that maximize the forces and velocities transmitted to the object, along a desired direction (task direction) [16]. Human trajectories are typically optimized for the task to be executed, but the process of mapping human to robot motion doesn't ensure that the extracted robot configurations will also be optimal for the desired task 1 . An optimization scheme can easily be formulated, that will derive task-specific configurations as well as task-specific, robust grasps for various robots arm hand systems. The problem of deriving taskspecific grasps has been studied in the past for both fully actuated and underactuated hands.

\footnotetext{
${ }^{1}$ Typically, low-complexity hands have different kinematic constraints and significant dimensional differences with the human hand.
} 
Typically, the task is represented modelling the wrench/twist transmission capabilities of a grasp with task ellipsoids. Li et al [17] first proposed a task-oriented quality measure, the maximization of which leads to task-specific grasps. An overview of other related studies focusing on grasp synthesis, can be found in [18]. In [19] and [20] different task compatibility indexes have been proposed. These indexes optimize taskspecificity of grasp configurations given specific task requirements in terms of wrenches and twists applied on the object. Recently, Boutselis et al [21], [22] proposed a methodology based on the concept of Q-distance [23] that derives taskspecific force closure grasps for fully actuated robot hands, under a wide range of uncertainties. Moreover, Mavrogiannis et al [24] proposed an optimization scheme that derives taskspecific force closure grasps for underactuated robot hands.

Regarding humanlike grasp planning, in [25] authors proposed Navigation Function models trained in a low-d manifold of human motion. These NF models used fictitious obstacle functions learned using B-Splines that: 1) embedded anthropomorphism and 2) applied repulsive effects on the robot artifact so as to attain new humanlike configurations. In [26] authors proposed the concept of "principal motion directions" to reduce the dimension of the search space in motion planning with anthropomorphic mechanical hands, avoiding collisions and mimicking real human hand postures. In [27] Ciocarlie et al, proposed an on-line grasp planner that utilizes a series of low-dimensional posture subspaces, in order to perform automated grasp synthesis with artificial hands, in real and simulated environments.

In this paper we propose a methodology for closed-loop, human-like, task-specific reaching and grasping with redundant arms and low-complexity hands. For doing so, we first formulate the human to robot motion mapping as a constrained non-linear optimization problem. Position and orientation goals are incorporated in the objective function together with a criterion of functional anthropomorphism [15], which is used to minimize structural dissimilarity between human and robot configurations. The derived humanlike trajectories are used to train Navigation Function models that generalize to new reach-to-grasp motions. In order to derive robust, task-specific grasps and task-specific arm configurations, we use specific grasp quality measures [28] and we optimize a set of task and grasp compatibility indexes (for the arm and the hand case), that maximize the velocity and force transmission ratios along the desired task directions. The final scheme provides: 1) anthropomorphic robot motion, 2) task specific robot arm configurations, 3 ) robust grasps and 4) guaranteed convergence to the desired goals.

The rest of the document is organized as follows: Section II presents the motion capture systems, the kinematic model of the human arm hand system, the robot arms and hands used, and the human data collection procedure, Section III discusses the various methods employed to formulate the proposed methodology, Section IV reports the results of the simulated and the experimental paradigms, while Section V concludes the paper.

\section{Apparatus, Models and Human Demonstrations}

\section{A. Motion Capture Systems}

In order to capture human arm kinematics the Liberty (Polhemus Inc.) magnetic motion capture system was used, which consists of a reference system and three magnetic position sensors. During the experiments, one sensor was placed on the human shoulder, one sensor on the human elbow and a third sensor on the human wrist. The Liberty system provides high accuracy for both position (0.03 inches) and orientation (0.15 degrees) at a frequency of $240 \mathrm{~Hz}$.

Human hand kinematics were gathered using the CyberGlove II (Cyberglove Systems) data-glove. The particular dataglove has 22 sensors that capture twenty DoF of the human hand and two DoF of the human wrist. More precisely, the flexion-extension of all three joints of each human finger, the abduction/adduction of the fingers, as well as the flexion/extension and the abduction/adduction of the wrist can be measured. The acquisition frequency of the Cyberglove II is $90 \mathrm{~Hz}$ and the accuracy is 1 degree.

\section{B. Kinematic Models of the Human Arm and Hand}

Human arm is modeled with 7 DoF. Three rotational DoF are used for the shoulder, one rotational DoF for the elbow, one rotational DoF for pronation/supination and two rotational DoF for the wrist. The human hand model is inspired by the way that Cyberglove II flex sensors are positioned. The model consists of fifteen joints and twenty DoF. Index, middle, ring and pinky fingers have three DoF for flexion/extension and one for abduction/adduction, while the thumb has two for flexion/extension, one for abduction/adduction and one to model thumb's ability to oppose to other fingers. Each finger is considered as an independent serial kinematic chain. A more sophisticated human hand model can be used in case there is a motion capture system available that can measure the extra DoF [29]. In order to derive the link lengths for the forward kinematics computations, we use parametric models derived from hand anthropometry studies [30].

\section{Robot Arms and Hands}

In this paper we provide a series of simulated and experimental paradigms using two robot arms and three robot hands. More precisely, the following robots are used: 1) the Barrett WAM 7 DoF robotic manipulator [31], 2) the Mitsubishi PA10 7 DoF robotic manipulator [32], 3) the OpenHand Model T42 two-fingered robot hand [3], 4) the iRobot-Harvard-Yale (iHY) three-fingered robot hand [33] and 5) the OpenBionics 4F fourfingered robot hand [4]. For simulations purposes the robot models were created using the Matlab Robotics Toolbox [34].

\section{Human Demonstrations}

In order to collect human demonstrations we conducted experiments with five healthy volunteers 21, 24, 27, 28 and 40 years old. All the experimental procedures were approved by the Institutional Review Board of the National Technical University of Athens and the subjects gave informed consent. 


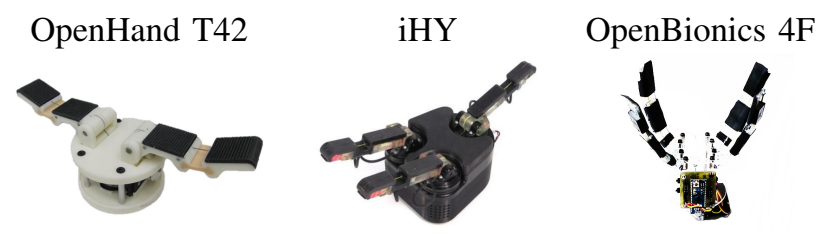

Barrett WAM Mitsubishi PA10

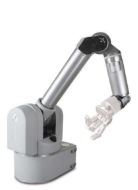

Fig. 1: The different robot arms and hands used.

All participants performed the experiments with their dominant arm (right arm for all cases) and they repeated multiple (10 per object) reaching movements to grasp a series of objects placed at five different positions in 3D space. A resting period of one minute was used between consecutive trials, in order for the subjects to avoid fatigue. Each subject executed multiple trials, for every object and object position combination.

\section{METHODS}

In this section, we present the different methods used to formulate the proposed methodology.

\section{A. Mapping Human to Humanlike Robot Arm Hand System Motion}

At first we formulate the problem of mapping human to humanlike robot motion, as a constrained non-linear optimization problem. We have experimentally validated that the problem is well formed and even when the algorithm terminates at local minimum, the solution suffices for our purposes. Such choice is typical for related studies [35].

1) Position and Orientation Metrics: Typically, robot hands may have non-anthropomorphic dimensions in terms of palm size, finger sizes, phalanges sizes, finger base frames coordinates etc. Thus, the application of common human to robot motion mapping methodologies like fingertips mapping, becomes difficult when human and robot wrist attain same position and orientation. In [15] we proposed to apply a wrist offset in order to compensate for dimensional differences between the human and robot hands. In this paper, we reformulate human to robot motion mapping in a unified manner, for the overall robot arm hand system. For doing so, we consider as system's end-effectors the robot fingertips which have to achieve same position and/or orientation with the human fingertips.

Let $\mathbf{x}_{R A H_{i}}=f_{R A H_{i}}\left(\mathbf{q}_{R A H_{i}}\right)$ denote the forward kinematics for each robot finger, let $m$ be the number of the fingers and $\mathbf{x}_{R A H_{i}}, \mathbf{x}_{\text {RAHgoal }} \in R^{3}$ denote the current and desired fingertip position respectively. We can define the following metric for position goals:

$$
P_{R A H}\left(\mathbf{q}_{R A H}\right)=\sum_{i=1}^{m}\left\|\mathbf{x}_{R A H_{i}}-\mathbf{x}_{\text {RAHgoal }}\right\|^{2}
$$

Regarding the orientation, let $\mathbf{h}_{c_{i}}=\left(a_{c_{i}}, b_{c_{i}}, c_{c_{i}}, d_{c_{i}}\right), \mathbf{h}_{g_{i}}=$ $\left(a_{g_{i}}, b_{g_{i}}, c_{g_{i}}, d_{g_{i}}\right) \in R^{4}$ denote the current and goal fingertip orientations (expressed using quaternions, to avoid singularities) for each finger. Then the distance in $\mathbb{S}^{3}$, between human and robot fingertip orientations is defined as:

$$
\bar{d}_{R A H o_{i}}\left(\mathbf{h}_{c_{i}}, \mathbf{h}_{g_{i}}\right)=\cos ^{-1}\left(a_{c_{i}} a_{g_{i}}+b_{c_{i}} b_{g_{i}}+c_{c_{i}} c_{g_{i}}+d_{c_{i}} d_{g_{i}}\right)
$$

Taking into account the antipodal points [36], we formulate the following distance metric for orientations:

$$
d_{R A H o_{i}}\left(\mathbf{h}_{c_{i}}, \mathbf{h}_{g_{i}}\right)=\min \left\{\bar{d}_{R A H o_{i}}\left(\mathbf{h}_{c_{i}}, \mathbf{h}_{g_{i}}\right), \bar{d}_{R A H o_{i}}\left(\mathbf{h}_{c_{i}},-\mathbf{h}_{g_{i}}\right)\right\}
$$

In this study, we have to map human to robot motion for robot hands that have less than five fingers. Previous studies used the virtual finger approach [37], computing a virtual fingertip position of the robot hand, as a linear combination of the fingertip positions of the human hand (e.g., ring and pinky fingers). In this paper, we assign human thumb fingertip position as a position goal for one of the robot fingers and then we use splines to calculate the remaining robot fingertip positions, interpolating between the other four positions of the rest human fingers and selecting $m-1$ equally distant points (where $m$ is the number of robot fingers). Spline is a low-degree polynomial function that is sufficiently smooth at the places where the polynomial curves connect (i.e., knots). Spline interpolation yields smaller errors than linear interpolation and the interpolant is smoother.

2) A Metric of Anthropomorphism: In this subsection we use a metric of anthropomorphism that diminishes structural dissimilarity between the human and the robot arm configurations [38]. Let $\mathbf{s}_{\text {elbow }} \in R^{3}$ be the human elbow position in 3D space and $S$ be the set of $n$ robot joint positions in $3 \mathrm{D}$ space. For $n$ points $\mathbf{s}_{1}, \mathbf{s}_{2}, \ldots, \mathbf{s}_{n}$, the distance between the human elbow and robot joints positions (excluding shoulder and end-effector), is given by:

$$
D=\sum_{j=1}^{n}\left\|\mathbf{s}_{j}-\mathbf{s}_{\text {elbow }}\right\|^{2}
$$

3) Problem Formulation: The objective function $F_{R A H}$ for the case of a robot arm hand system, under position, orientation and anthropomorphism goals, becomes:

$$
\begin{array}{r}
F_{R A H}\left(\mathbf{q}_{R A H}\right)=w_{R A H x} P_{R A H}\left(\mathbf{q}_{R A H}\right)+ \\
w_{R A H o} \sum_{i=1}^{m} d_{R A H o_{i}}\left(\mathbf{h}_{c_{i}}, \mathbf{h}_{g_{i}}\right)+w_{D} D
\end{array}
$$

where $w_{R A H x}, w_{R A H o}$ and $w_{D}$ are the weights that adjust the relative importance of the position, orientation and anthropomorphism terms respectively and $D$ is the sum of distances between the human elbow and the robot joint positions. Hence, the mapping problem is in this case formulated as follows:

$$
\text { minimize } F_{R A H}\left(\mathbf{q}_{R A H}\right)
$$

subject to the inequality constraints of joints limits:

$$
\mathbf{q}_{R A H}^{-}<\mathbf{q}_{R A H}<\mathbf{q}_{R A H}^{+}
$$


where $\mathbf{q}_{R A H}$ is the vector of the joint angles of the robot arm hand system and $\mathbf{q}_{R A H}^{-}, \mathbf{q}_{R A H}^{+}$are the lower and upper limits respectively. Moreover, for each finger we may also adopt equality constraints that encapsulate possible joint couplings. For solving the optimization problem the fmincon function of MATLAB was used, with the interior-point algorithm. A review of different metrics and methods can be found in [38].

It must be noted that in this paper we introduce task goals via position and orientation metrics in the objective function and not as equality constraints, because otherwise for many cases the problem would become infeasible. Using such an approach, the user is able to select the position and orientation accuracy (appropriately defining the corresponding weights), which may be lower for free space motions (focusing on anthropomorphism) and very high during grasping or any other interaction with the environment.

\section{B. Training Navigation Function Models}

Following the aforementioned procedure we manage to map human to anthropomorphic robot motion for a complete robot arm hand system and derive a series of humanlike reach to grasp robot joint-space trajectories. These trajectories can be used in order to train Navigation Function (NF) models that learn new anthropomorphic robot motions and generalize to new grasping tasks. The Navigation Functions models were first proposed by Rimon and Koditschek [39], [40] and some of their characteristics are: 1) they provide closed-loop motion planning, 2) they guarantee convergence to the desired goals, 3) they have highly nonlinear learning capability, 4) they provide continuous and smooth trajectories, 5) they embed anthropomorphism (synthesizing "fictitious" obstacle functions) and finally 6) they can generalize for similar, neighboring configurations (goal configurations).

In this paper we train NF models that use "fictitious" obstacle functions learned in the low dimensional space of the anthropomorphic robot kinematics. These functions apply repulsive effects on the robot arm hand system, so as to reach anthropomorphic configurations. In order to learn the structure of the NF obstacle functions, we use B-Splines as described in [25]. The control law is constructed as follows:

$$
u(t)=-\mathbf{K}_{p} \nabla_{q} \phi\left(\mathbf{x}_{t}\right)
$$

where $\mathbf{x}_{t}$ is the system state and $\phi$ is the navigation function responsible to: 1) drive the system to its final configuration and 2) generate new anthropomorphic robot trajectories (similar to those used during training). $\mathbf{K}_{p}>0$ is a constant gain matrix. The navigation function is given by:

$$
\phi=\frac{\gamma_{d}(\mathbf{q})}{\left(\gamma_{d}^{k}(\mathbf{q})+\beta\right)^{\frac{1}{k}}}
$$

where $\mathbf{q}, \mathbf{q}_{d}$ are the current the desired configurations respectively, $\gamma_{d}(\mathbf{q})=\left\|\mathbf{q}-\mathbf{q}_{d}\right\|^{2}$ is the paraboloid attractive effect, $\beta=\prod_{i \in I_{0}} \beta_{i}$ is the aggregated obstacle repulsive effects and $k \in N \backslash\{0,1\}$ is a tuning parameter. More details regarding the NF, can be found in [25].
In this paper we follow a similar approach with [25], training the NF models with the anthropomorphic robot motion (joint space trajectories) instead of the human motion. More precisely, for the training of the NF models we use the humanlike robot motion that we derived from the aforementioned human to robot motion mapping scheme. The extracted humanlike robot data are represented in a lower dimensional manifold using the Principal Components Analysis (PCA) The output of the NF models, is back-projected in the high dimensional space in order to control the robot arm hand system. In this case, no online human to robot motion mapping is required, thus computational effort diminishes (all computations are performed offline). All models require as input the "goal" position in the low-d space of the humanlike robot kinematics.

\section{Deriving Task-Specific Robot Arm Configurations and Task-Specific, Robust Grasps}

In this section we derive task-specific robot arm configurations and task-specific, robust grasps that will be fed to the NF models as new goals.

1) Deriving Task-Specific Robot Arm Configurations: It is well known that at any posture the velocity and force transmission characteristics of a manipulator can be geometrically represented as ellipsoids. Let $\mathbf{J}$ be the manipulator Jacobian that maps the joint space velocities $\dot{\mathbf{q}}$ to task space velocities $\dot{\mathbf{x}}$ with $\dot{\mathbf{x}}=\mathbf{J}(\mathbf{q}) \dot{\mathbf{q}}$ and the joint space torques $\tau$ to task space forces $\mathbf{f}$ with $\tau=\mathbf{J}^{T}(\mathbf{q}) \mathbf{f}$. The velocity (or manipulability) ellipsoid is defined as:

$$
\dot{\mathbf{x}}^{T}\left(\mathbf{J J}^{T}\right)^{-1} \dot{\mathbf{x}} \leq 1
$$

while the force ellipsoid is defined as:

$$
\mathbf{f}^{T}\left(\mathbf{J J}^{T}\right) \mathbf{f} \leq 1
$$

According to Yoshikawa [41] when you maximize the volume of the manipulability ellipsoid you move to configurations away from singularities. The volume of the ellipsoid is proportional to the quantity:

$$
V_{M E}(\mathbf{q})=\sqrt{\operatorname{det}\left(\mathbf{J}(\mathbf{q}) \mathbf{J}^{T}(\mathbf{q})\right)}
$$

This quantity is called the "Manipulability Measure" and expresses the ability of the end effector to move or exert forces to a random direction. In order to maximize the capability of a robot arm to move fastly or exert forces at a specific direction you have to maximize appropriately the force or velocity transmission ratios along this direction. Let $\mathbf{d}$ denote the unit vector in the direction of interest and $\alpha$ the distance along the d vector from the origin to the surface of the ellipsoid. The scalar $\alpha$ is the force transmission ratio in the direction of $\mathbf{d}$. But $\alpha \mathbf{d}$ is also a point on the ellipsoid surface and must satisfy the following equation:

$$
(\alpha \mathbf{d})^{T}\left(\mathbf{J J}^{T}\right)(\alpha \mathbf{d})=1
$$

\footnotetext{
${ }^{2}$ The first 3 principal components extracted using the PCA method account for more than $88 \%$ of the data variance for both the arm and the hand case.
} 
Thus, we derive the force transmission ratio $\alpha$, as follows:

$$
\alpha^{a}=\left[\mathbf{d}^{T}\left(\mathbf{J} \mathbf{J}^{T}\right) \mathbf{d}\right]^{-1 / 2}
$$

Through a similar derivation, the velocity transmission ratio $\beta$ in the direction of $\mathbf{d}$ is defined as:

$$
\beta^{a}=\left[\mathbf{d}^{T}\left(\mathbf{J J}^{T}\right)^{-1} \mathbf{d}\right]^{-1 / 2}
$$

Now we can define the task compatibility index for a task described by $m$ task coordinates. Let $\mathbf{d}_{i}$ (with $i=1,2, \ldots l$ ) be the direction vectors across which we want to control force and let $\mathbf{d}_{j}$ (with $\mathrm{j}=1+1,1+2, \ldots \mathrm{m}$ ) be the direction vectors across which we want to control velocity. Thus, we denote $\alpha_{i}^{a}$ the force transmission ratio in the direction of $\mathbf{d}_{i}$ and $\beta_{j}^{a}$ the velocity transmission ratio in the direction of $\mathbf{d}_{j}$. The task compatibility index, is defined as:

$$
T C=\frac{1}{\left(\alpha_{1}^{a} \alpha_{2}^{a} \ldots \alpha_{l}^{a}\right)\left(\beta_{l+1}^{a} \beta_{l+2}^{a} \ldots \beta_{m}^{a}\right)}
$$

2) Deriving Task-Specific Grasps: In a similar fashion, we define a grasping task by it's requirements in wrench/twist transmission to the object. Thus, for robot hands we optimize a grasp-compatibility index similar to the index defined for the robot arm. The velocity transmission ratio expresses how much a unitary change of the joint angle velocities affects the twist on the object's center of mass, while the force transmission ratio expresses how much a unitary change of the joint torques affects the wrench on the object's center of mass. The maximization of the transmission ratios can be geometrically represented as an alignment between the principal axis of the corresponding ellipsoid and task direction d. Thus, for the hand case the force and velocity transmission ratios become:

$$
\begin{gathered}
\alpha^{h}=\left[\mathbf{d}^{T}\left(\mathbf{H}(\mathbf{q}) \mathbf{H}^{T}(\mathbf{q})\right) \mathbf{d}\right]^{-1 / 2} \\
\beta^{h}=\left[\mathbf{d}^{T}\left(\mathbf{H}(\mathbf{q}) \mathbf{H}^{T}(\mathbf{q})\right)^{-1} \mathbf{d}\right]^{-1 / 2}
\end{gathered}
$$

where $\mathbf{H}$ is the hand-object Jacobian and $\alpha^{h}$ and $\beta^{h}$ are the hand force and velocity transmission ratios respectively. In order to achieve a task-specific grasp wrt a task's requirements in force and velocity transmission and control, we have to appropriately maximize the aforementioned ratios. The grasp compatibility index is defined similarly to (16):

$$
G C=\frac{1}{\left(\alpha_{1}^{h} \alpha_{2}^{h} \ldots \alpha_{l}^{h}\right)\left(\beta_{l+1}^{h} \beta_{l+2}^{h} \ldots \beta_{m}^{h}\right)}
$$

3) Utilizing Grasping Quality Measures: In order to achieve robust grasps, we introduce specific grasp quality measures in our objective function. Two different measures based on geometric relations [28] are considered for the different hands: 1) the distance between the centroid of the contact polygon and the object geometric centroid and 2) the area of the grasp polygon. a) Distance between the contact polygon centroid and the geometric centroid of the object: In order to minimize the effect of inertial and gravitational forces on the grasp, we have to minimize the distance between the object's geometric centroid $\left(C_{G}\right)$ and the contact polygon's centroid $\left(C_{C}\right)$. Thus the metric becomes:

$$
Q_{D C}=\left\|C_{G}-C_{C}\right\|
$$

b) Area of the grasp polygon: In order to derive a robust grasp that resists large disturbances with the same contact forces we have to maximize the area of the grasp polygon. A metric that computes this area for the simple case of three contact points (e.g., iHY is a three fingered hand), is defined as follows:

$$
Q_{A G P}=\operatorname{Area}\left(\text { Triangle }\left(\boldsymbol{c}_{1}, \boldsymbol{c}_{2}, \boldsymbol{c}_{\mathbf{3}}\right)\right)
$$

where $c_{1}, c_{2}, c_{3}$ are contact points on the object surface.

4) Problem Formulation: Thus, we need to perform a constrained minimization of the following objective function:

$$
\mathrm{G}(\mathbf{p})=w_{T C} T C+w_{G C} G C+w_{Q} Q+w_{D} D
$$

wrt the decision variables

$$
\mathbf{p}=\left[\begin{array}{ll}
\mathbf{q}_{R A}^{T} & \mathbf{q}_{R H}^{T}
\end{array}\right]^{T} \in R^{n_{q r a}+n_{q r h}}
$$

where $n_{q r a}, n_{q r h}$ are the numbers of the robot arm and robot hand joint variables respectively, $Q$ is the value of the selected grasp quality metrid ${ }^{3}$ and $w_{T C}, w_{G C}, w_{Q}, w_{D}$ are the weights that adjust the relative importance of the task compatibility, grasp compatibility, grasp quality measure and anthropomorphism ${ }^{4}$ terms respectively. Thus, the optimization problem is formulated as follows:

$$
\mathbf{p}^{*}=\underset{\mathbf{p}}{\operatorname{argmin}} \mathbf{G}(\mathbf{p})
$$

s.t.

$$
\begin{gathered}
\mathbf{q}_{R A H}^{-}<\mathbf{q}_{R A H}<\mathbf{q}_{R A H}^{+} \\
\mathbf{q}_{i}=k_{i} \mathbf{q}_{i-1} \\
\text { fkine }\left(\mathbf{q}_{R A H}\right) \in \partial O \\
\mathbf{q}_{\text {abd/add }}^{i} \leq \mathbf{q}_{\text {abd/add }}^{i+1} \\
\mathbf{S}_{l} \notin O
\end{gathered}
$$

where (25) denotes the joint limit constraints, 26) denotes the weighted by $k_{i}$ coupling between specific joints of robot hands, 27p bounds the fingertips to lie on the object's surface, 28) avoids abduction/adduction collisions for the iHY robot hand and 29) guarantees that no point on the robot hand links (in discrete set $S_{l}$ ) will penetrate the object.

\footnotetext{
${ }^{3} Q$ can be defined by eq. 20 or 21 and depends on the chosen robot hand.

${ }^{4}$ In order to compute the value of the metric of anthropomorphism, we use as reference the elbow position of the human configuration that reaches closer to the desired object position (closer to the wrist).
} 


\section{Methodology Outline}

In order to formulate the proposed methodology, we use the following steps:

- Human demonstrations (human motion data) are collected for the tasks to be executed.

- Human motion is transformed to anthropomorphic robot motion using the aforementioned mapping scheme.

- A low-d manifold of the anthropomorphic robot motion is derived using Principal Components Analysis (PCA).

- Navigation Function (NF) models are trained in the low-d space of the anthropomorphic robot motion.

- Task-specific robot configurations and task-specific, robust grasps are derived using appropriate task and grasp compatibility indexes and grasp quality measures.

- The derived task-specific robot configurations are used as goal configurations for the NF models, that produce new humanlike robot motion.

- The produced humanlike robot motion is back projected to the high-d space, to control the arm hand system.

- Navigation Function models guarantee convergence to the desired goals, efficiently executing the required task.

\section{Results}

In this section we validate the efficiency of the proposed methods, using a series of simulated and experimental paradigms with different robot arm hand systems. In Fig. 2 we present instances of three different trajectories executed with two different robot arm hand systems. The first example depicted in subfigure 2a) focuses only on trajectory tracking (in task space) while the other two trajectories optimize also anthropomorphism of robot configurations. The robot elbow is much closer to the human elbow for the anthropomorphic results, as structural dissimilarity between human and robot configurations has been minimized. The reason that the human and robot elbow positions are not coincident is owed to the huge dimensional differences (in terms of link lengths) between the human and the robot arm.

Fig. 3 presents the effect of the task compatibility index metric in the derived anthropomorphic configuration. As it can be noticed the robot arm posture is slightly changed, in order for the major axis of the velocity ellipsoid to be aligned with the task direction. In Fig. 4 the Barrett WAM + i-HY robot arm hand system grasps a spherical object. All robot hand constraints are guaranteed and the task compatibility index of the robot arm hand system is optimized. More specifically in subfigure 4a) the force transmission ratio is maximized across a specific direction while in $4 b$ ) the velocity transmission ratio is maximized along the same direction.

Finally, in Fig. 5 two different grasps with the OpenHand T42 and a cylindrical object are depicted. Subfigure 5a), presents a random grasp satisfying that the contact points will lie on the object surface and that no robot link will penetrate the object. Subfigure 5b) presents an optimal grasp wrt the grasping quality metric proposed in eq. 20 and which has a minimized distance between the object's geometric centroid and the contact polygon's centroid.

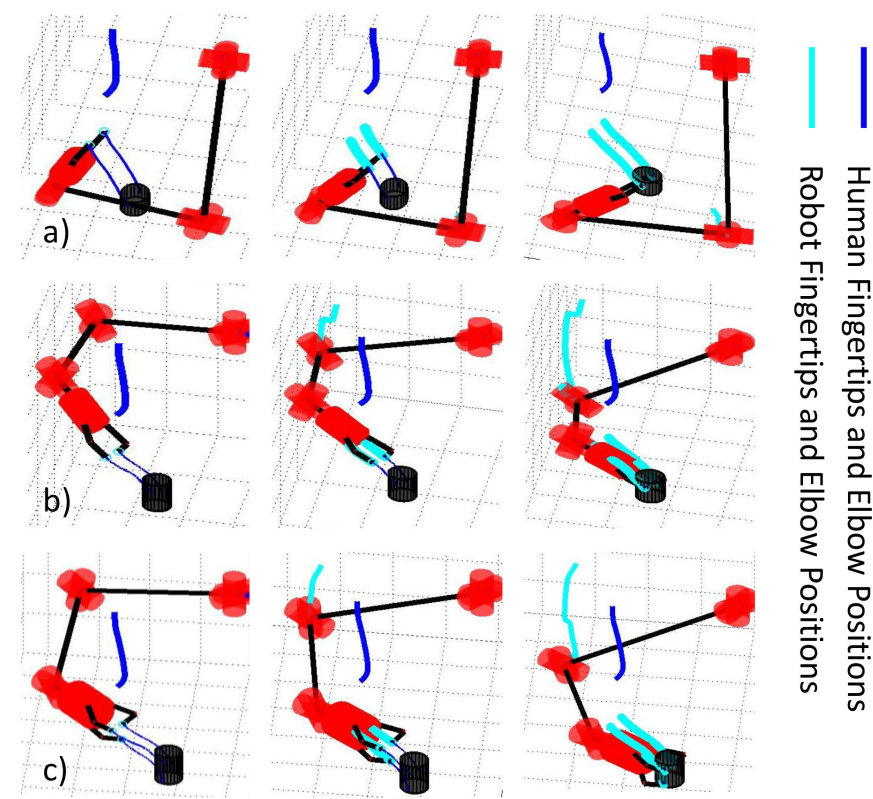

Fig. 2: Deriving humanlike trajectories for reach to grasp movements. Row a) presents instances of the trajectory executed with the Barrett WAM arm and the OpenHand T42 robot hand without a criterion of anthropomorphism. Row b) presents instances of the trajectory executed with the same robot arm hand system, using the criterion of functional anthropomorphism. Row c) presents instances of the trajectory executed with the Barrett WAM robot arm and the iHY robot hand, using a criterion of functional anthropomorphism. Human fingertips and elbow positions are represented by the blue lines, while robot fingertips and elbow positions are represented by the cyan lines.

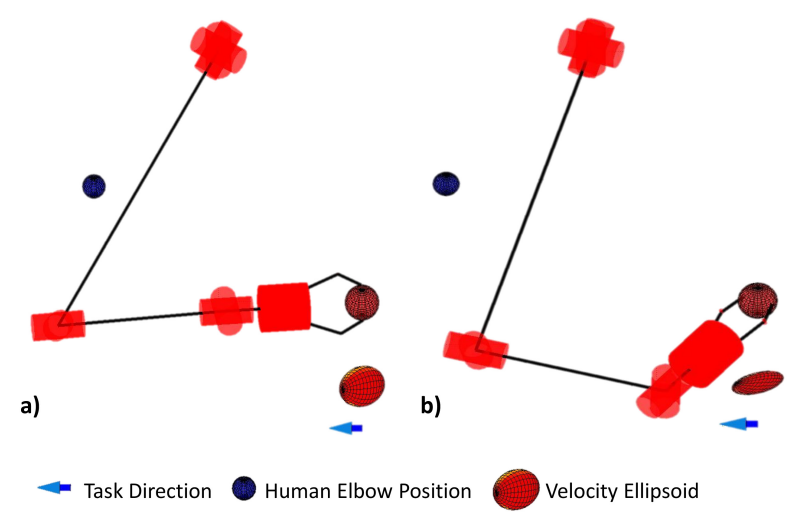

Fig. 3: The Barrett WAM + OpenHand T42 robot arm hand system, is used to grasp a spherical object using an anthropomorphic configuration (employing a metric of anthropomorphism to minimize structural dissimilarity between human and robot arms). Subfigure a) presents the derived configuration without employing the task compatibility index (velocity ellipsoid is almost spherical). Subfigure b) presents the robot arm hand system's configuration after the incorporation of the task compatibility index criterion. Velocity transmission ratio is maximized and the major axis of the manipulability ellipsoid is aligned with task direction. The blue sphere represents human elbow position (the distance between human and robot elbow is minimized). 


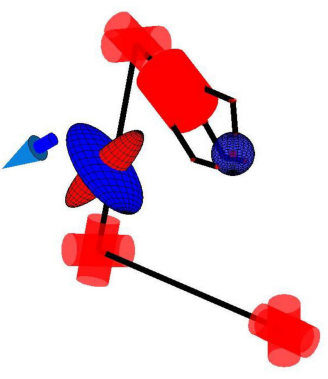

a)

Task Direction

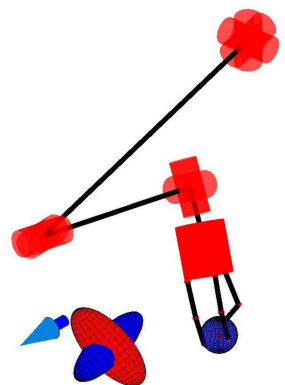

b)
Fig. 4: The Barrett WAM + i-HY robot arm hand system, is used to grasp a spherical object maximizing force and velocity transmission ratios along a specific task direction. The criterion of anthropomorphism is omitted in order to highlight the differences between the optimal configurations for the different task specifications. For all cases, the major axis of the "desired" ellipsoid (velocity or force) is aligned with the task direction and the corresponding transmission ratio is maximized.

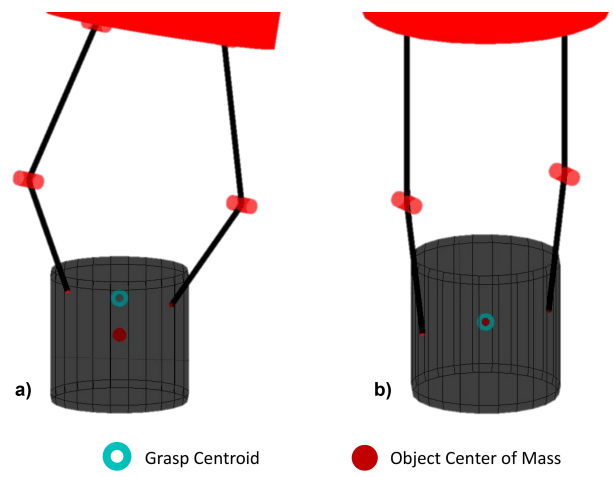

Fig. 5: The OpenHand T42 is used to grasp a cylindrical object. Subfigure a) presents a random grasp, while subfigure b) presents an optimal grasp for which the distance between the object's geometric centroid $\left(C_{G}\right)$ and the contact polygon's centroid $\left(C_{C}\right)$ is minimized. For both cases all robot hand constraints are satisfied.
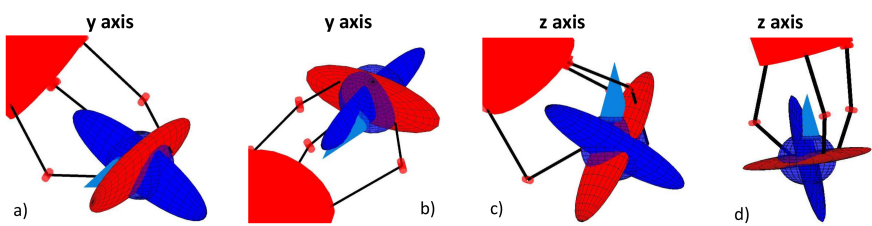

a Task Direction

Force Ellipsoid

Velocity Ellipsoid

Fig. 6: The iHY robot hand is used to grasp a spherical object in a task specific manner optimizing a task-compatibility index along the desired directions. Subfigures a) and b) depict grasps maximizing the force and velocity transmission ratios respectively, along the y axis. Subfigures c) and d) depict grasps maximizing the force and velocity transmission ratios respectively, along the $\mathrm{z}$ axis. For all cases the major axis of the "desired" ellipsoid (velocity or force) aligns with the desired task direction.
In Fig. 6 we present grasps of a spherical object with the iHY hand for which the task compatibility index is optimized (maximizing force and velocity transmission ratios along the desired directions). It must be noted that a large value of the $G C$ index indicates good control compatibility (finer control of force and velocity can be achieved) while a small value indicates good compatibility for effecting large forces and velocities in the respective directions. Thus choice of directions and the maximization or minimization of the index depends on the task requirements.

The accompanying video presents an experiment with the Mitsubishi PA + OpenBionics 4F robot arm hand system repeatedly reaching in a humanlike fashion and grasping a wide range of everyday life objects [42] with a specific preplanned grasp. As it can be noticed the low-complexity, compliant hand facilitates grasping even under object position uncertainties (when the object is perturbed or when the object position is not accurately known). Images of the conducted experiment are depicted in Fig. 7

\section{CONCLusions}

In this paper, we proposed a methodology for closed-loop, humanlike, task-specific reaching and grasping with redundant robot arms and low-complexity robot hands. Human demonstrations are utilized to map human to anthropomorphic robot motion. Principal Components Analysis is used to represent the humanlike robot motion in a low-dimensional manifold where appropriate path planning models are trained. The proposed scheme provides: 1) anthropomorphic robot arm trajectories, 2) task-specific robot arm and hand configurations, maximizing the task and grasp compatibility indexes of the arm and hand respectively, 3) robust grasps, utilizing appropriate grasp quality measures and 4) guaranteed convergence to the desired goals. In this work we consider the position and geometry of objects a-priori known.

\section{REFERENCES}

[1] R. Ma and A. Dollar, "On dexterity and dexterous manipulation," in 15th International Conference on Advanced Robotics (ICAR), June 2011, pp. $1-7$.

[2] A. M. Dollar and R. D. Howe, "The highly adaptive SDM hand: Design and performance evaluation," The International Journal of Robotics Research, 2010.

[3] R. Ma, L. Odhner, and A. Dollar, "A modular, open-source 3D printed underactuated hand," in IEEE International Conference on Robotics and Automation (ICRA), May 2013, pp. 2737-2743.

[4] A. G. Zisimatos, M. V. Liarokapis, C. I. Mavrogiannis, and K. J. Kyriakopoulos, "Open-source, affordable, modular, light-weight, underactuated robot hands," in IEEE/RSJ International Conference on Intelligent Robots and Systems (IROS), September 2014.

[5] F. Hegel, S. Krach, T. Kircher, B. Wrede, and G. Sagerer, "Understanding social robots: A user study on anthropomorphism," in IEEE International Symposium on Robot and Human Interactive Communication, August 2008, pp. 574-579.

[6] B. R. Duffy, "Anthropomorphism and the social robot," Robotics and Autonomous Systems, vol. 42, no. 3-4, pp. 177-190, 2003.

[7] J. Hong and X. Tan, "Calibrating a VPL Dataglove for teleoperating the Utah/MIT hand," in IEEE International Conference on Robotics and Automation (ICRA), vol. 3, 1989, pp. 1752-1757.

[8] T. H. Speeter, "Transforming human hand motion for telemanipulation," Presence: Teleoper. Virtual Environ., vol. 1, no. 1, pp. 63-79, January 1992. 

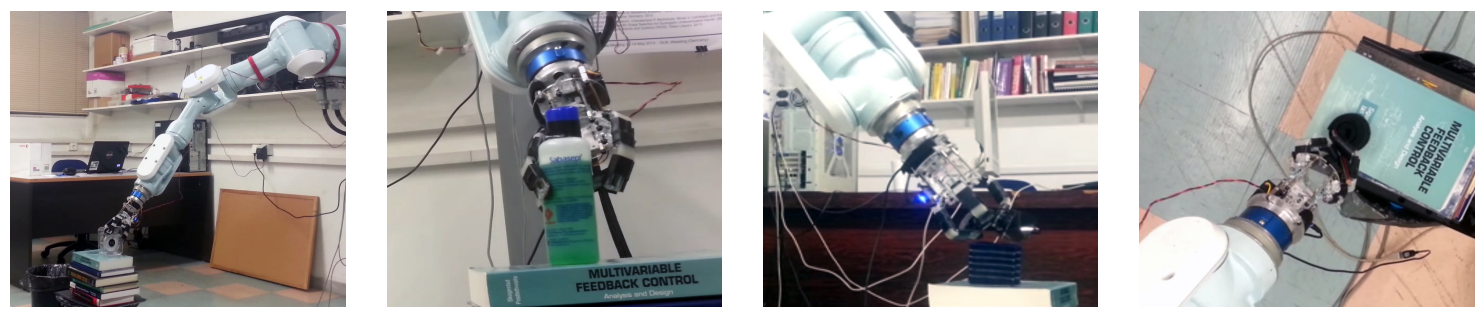

Fig. 7: Instances of the experiment conducted with the Mitsubishi PA10 + OpenBionics 4F robot arm hand system. The robot is depicted grasping: a plastic timer, a bottle full of liquid, a pair of sunglasses and a pencil.

[9] M. Ciocarlie, C. Goldfeder, and P. Allen, "Dexterous grasping via eigengrasps: A low-dimensional approach to a high-complexity problem," in Robotics: Science and Systems Manipulation Workshop - Sensing and Adapting to the Real World, 2007.

[10] L. Pao and T. Speeter, "Transformation of human hand positions for robotic hand control," in IEEE International Conference on Robotics and Automation (ICRA), vol. 3, May 1989, pp. 1758-1763.

[11] W. B. Griffin, R. P. Findley, M. L. Turner, and M. R. Cutkosky, "Calibration and mapping of a human hand for dexterous telemanipulation," in ASME IMECE 2000 Symposium on Haptic Interfaces for Virtual Environments and Teleoperator Systems, 2000.

[12] D. Tolani, A. Goswami, and N. I. Badler, "Real-time inverse kinematics techniques for anthropomorphic limbs," Graph. Models Image Process., vol. 62, no. 5, pp. 353-388, September 2000.

[13] F. Zacharias, C. Schlette, F. Schmidt, C. Borst, J. Rossmann, and G. Hirzinger, "Making planned paths look more human-like in humanoid robot manipulation planning," in IEEE International Conference on Robotics and Automation (ICRA), may 2011, pp. $1192-1198$.

[14] S. Albrecht, K. Ramirez-Amaro, F. Ruiz-Ugalde, D. Weikersdorfer, M. Leibold, M. Ulbrich, and M. Beetz, "Imitating human reaching motions using physically inspired optimization principles," in 11th IEEE-RAS International Conference on Humanoid Robots (Humanoids), oct. 2011, pp. $602-607$.

[15] M. V. Liarokapis, P. K. Artemiadis, and K. J. Kyriakopoulos, "Functional anthropomorphism for human to robot motion mapping," in 21st IEEE International Symposium on Robot and Human Interactive Communication (RO-MAN), Sept. 2012, pp. 31-36.

[16] J. Friedman and T. Flash, "Task-dependent selection of grasp kinematics and stiffness in human object manipulation," Cortex, vol. 43, no. 3, pp. $444-460,2007$.

[17] Z. Li and S. Sastry, "Task-oriented optimal grasping by multifingered robot hands," IEEE Journal of Robotics and Automation, vol. 4, no. 1, pp. 32-44, Feb 1988.

[18] A. Sahbani, S. El-Khoury, and P. Bidaud, "An overview of 3d object grasp synthesis algorithms," Robotics and Autonomous Systems, vol. 60, no. 3, pp. 326 - 336, 2012, autonomous Grasping.

[19] Q. Liu, "A task compatibility index for multi-fingered robot hand grasp planning," in Advanced Design and Manufacture to Gain a Competitive Edge, X.-T. Yan, C. Jiang, and B. Eynard, Eds. Springer London, 2008, pp. 653-661.

[20] S. L. Chiu, "Task compatibility of manipulator postures," The International Journal of Robotics Research, vol. 7, no. 5, pp. 13-21, 1988.

[21] G. I. Boutselis, C. P. Bechlioulis, M. V. Liarokapis, and K. J. Kyriakopoulos, "An integrated approach towards robust grasping with tactile sensing," in IEEE International Conference on Robotics and Automation (ICRA), May 2014, pp. 3682-3687.

[22] G. Boutselis, C. Bechlioulis, M. Liarokapis, and K. Kyriakopoulos, "Task specific robust grasping for multifingered robot hands," in IEEE/RSJ International Conference on Intelligent Robots and Systems (IROS), Sept 2014, pp. 858-863.

[23] X. Zhu and J. Wang, "Synthesis of force-closure grasps on 3-d objects based on the q distance," IEEE Transactions on Robotics and Automation, vol. 19, no. 4, pp. 669-679, Aug 2003.

[24] C. I. Mavrogiannis, C. P. Bechlioulis, M. V. Liarokapis, and K. J. Kyriakopoulos, "Task-specific grasp selection for underactuated hands," in IEEE International Conference on Robotics and Automation (ICRA), May 2014, pp. 3676-3681.
[25] I. Filippidis, K. Kyriakopoulos, and P. Artemiadis, "Navigation functions learning from experiments: Application to anthropomorphic grasping," in IEEE International Conference on Robotics and Automation (ICRA), may 2012 , pp. $570-575$.

[26] J. Rosell, R. Suárez, C. Rosales, and A. Pérez, "Autonomous motion planning of a hand-arm robotic system based on captured human-like hand postures," Autonomous Robots, vol. 31, no. 1, pp. 87-102, 2011.

[27] M. T. Ciocarlie and P. K. Allen, "Hand posture subspaces for dexterous robotic grasping," The International Journal of Robotics Research, vol. 28 , no. 7, pp. 851-867, 2009.

[28] M. Roa and R. Surez, "Grasp quality measures: review and performance," Autonomous Robots, pp. 1-24, 2014.

[29] M. V. Liarokapis, P. K. Artemiadis, and K. J. Kyriakopoulos, "Quantifying anthropomorphism of robot hands," in IEEE International Conference on Robotics and Automation (ICRA), 2013, pp. 2041-2046.

[30] B. Buchholz, T. J. Armstrong, and S. A. Goldstein, "Anthropometric data for describing the kinematics of the human hand," Ergonomics, vol. 35, no. 3, pp. 261-273, 1992.

[31] F. Smith and B. Rooks, "The harmonious robot," Industrial Robot: An International Journal, vol. 33, no. 2, pp. 125-130, 2006.

[32] N. Bompos, P. Artemiadis, A. Oikonomopoulos, and K. Kyriakopoulos, "Modeling, full identification and control of the mitsubishi PA-10 robot arm," in IEEE/ASME International Conference on Advanced Intelligent Mechatronics (AIM), Sept 2007, pp. 1-6.

[33] L. U. Odhner, L. P. Jentoft, M. R. Claffee, N. Corson, Y. Tenzer, R. R. Ma, M. Buehler, R. Kohout, R. D. Howe, and A. M. Dollar, "A compliant, underactuated hand for robust manipulation," The International Journal of Robotics Research, vol. 33, no. 5, pp. 736-752, 2014.

[34] P. Corke, "MATLAB toolboxes: robotics and vision for students and teachers," IEEE Robotics Automation Magazine, vol. 14, no. 4, pp. 1617, dec 2007.

[35] J. Zhao and N. I. Badler, "Inverse kinematics positioning using nonlinear programming for highly articulated figures," ACM Trans. Graph., vol. 13, no. 4, pp. 313-336, Oct. 1994.

[36] S. M. LaValle, Planning Algorithms. Cambridge, U.K.: Cambridge University Press, 2006, available at http://planning.cs.uiuc.edu/.

[37] M. Arbib, T. Iberall, and D. Lyons, "Coordinated control programs for movements of the hand," Experimental brain research, pp. 111-129, 1985.

[38] M. V. Liarokapis, P. K. Artemiadis, C. P. Bechlioulis, and K. J. Kyriakopoulos, "Directions, methods and metrics for mapping human to robot motion with functional anthropomorphism: A review," School of Mechanical Engineering, National Technical University of Athens, Tech. Rep., September 2013.

[39] D. Koditschek and E. Rimon, "Robot navigation functions on manifolds with boundary," Advances in Applied Mathematics, vol. 11, no. 4, pp. 412-442, 1990.

[40] E. Rimon and D. Koditschek, "Exact robot navigation using artificial potential fields," IEEE Transactions on Robotics and Automation, vol. 8, no. 5, pp. 501-518, 1992.

[41] T. Yoshikawa, "Analysis and Control of Robot Manipulators with Redundancy," in The First International Symposium on Robotics Research, M. Brady and R. Paul, Eds. MIT Press, 1984, pp. 735-747.

[42] https://www.youtube.com/watch? $\mathrm{v}=1 \mathrm{j} \_\mathrm{t} J 2 \mathrm{KIy}-\mathrm{c}$ 